\title{
Peritoneal Carcinomatosis: unravelling its main features and mimics
}

Rebelo, João; Sousa, Célia; Moreira, Adriana; Leitão, Patrícia; Preto, Ana Sofia

\section{Background}

Peritoneal carcinomatosis is the intraperitoneal dissemination of any tumour that does not originate from the peritoneum itself.

$t$ is the most common diffuse peritonea disease

Malignant and benign diseases use these same conduits to spread throughout the abdomen and pelvis
Secondary peritoneal malignancies far outnumber primary peritoneal neoplasms, such as peritoneal mesothelioma.

\section{Imaging Findings}

The hallmark of peritoneal carcinomatosis is presence of peritoneal thickening and omental cake.
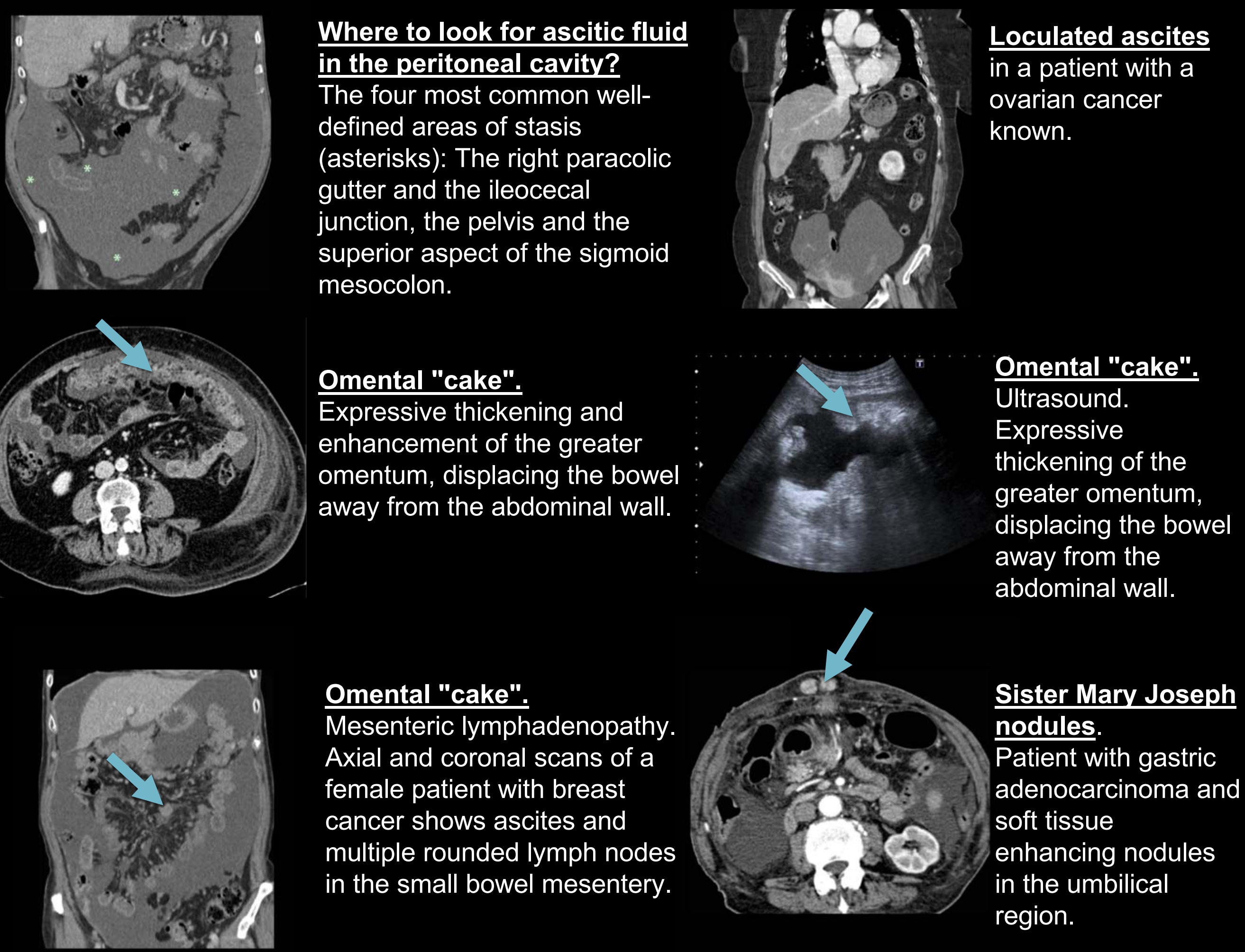

\section{Differential Diagnosis}

Benign etiologies may mimic peritoneal

carcinomatosis such as in cases of disseminated peritoneal endometriosis, leiomyomatosis, and

tuberculosis.

\section{Pseudomyxoma peritonei.}

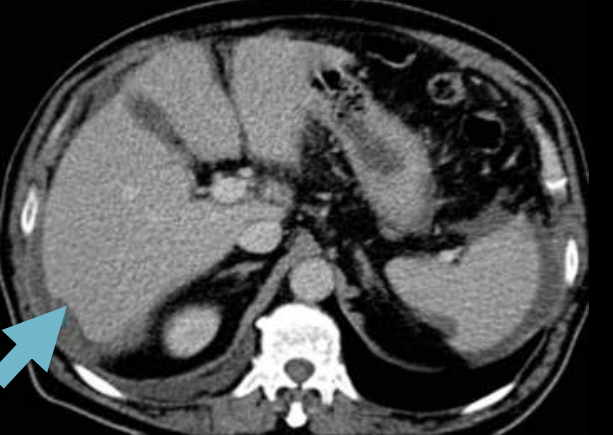

mall amount of perihepatic and perisplenic fluid, with mass effect (scalloping) on the posterior surface of the liver (arrow), characteristic of pseudomyxoma peritonei.

\section{Malignant Mesothelioma.}
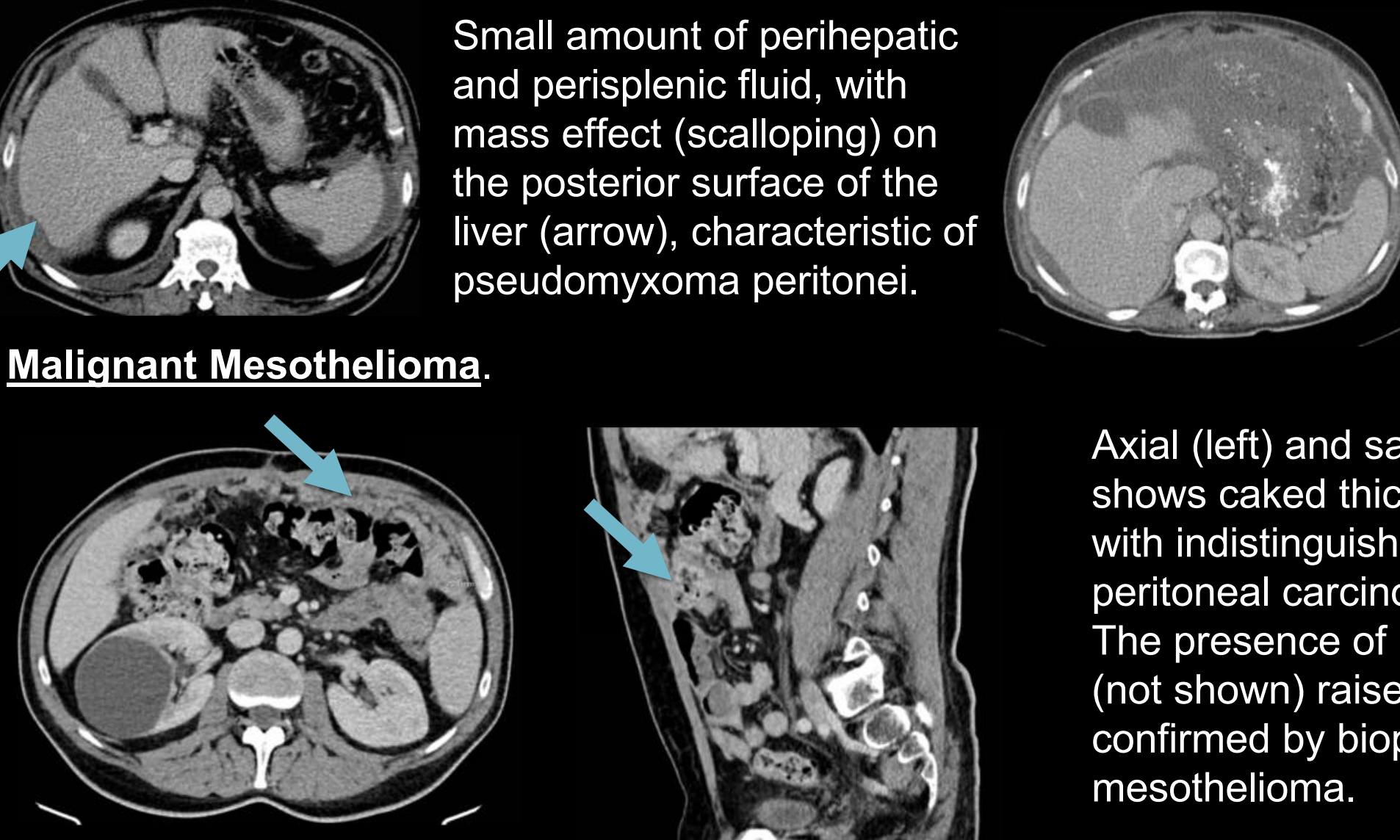

Ascitis with punctiform calcifications in a case of mucinous gastric adenocarcinoma.

Diffuse peritoneal leiomyomatosis
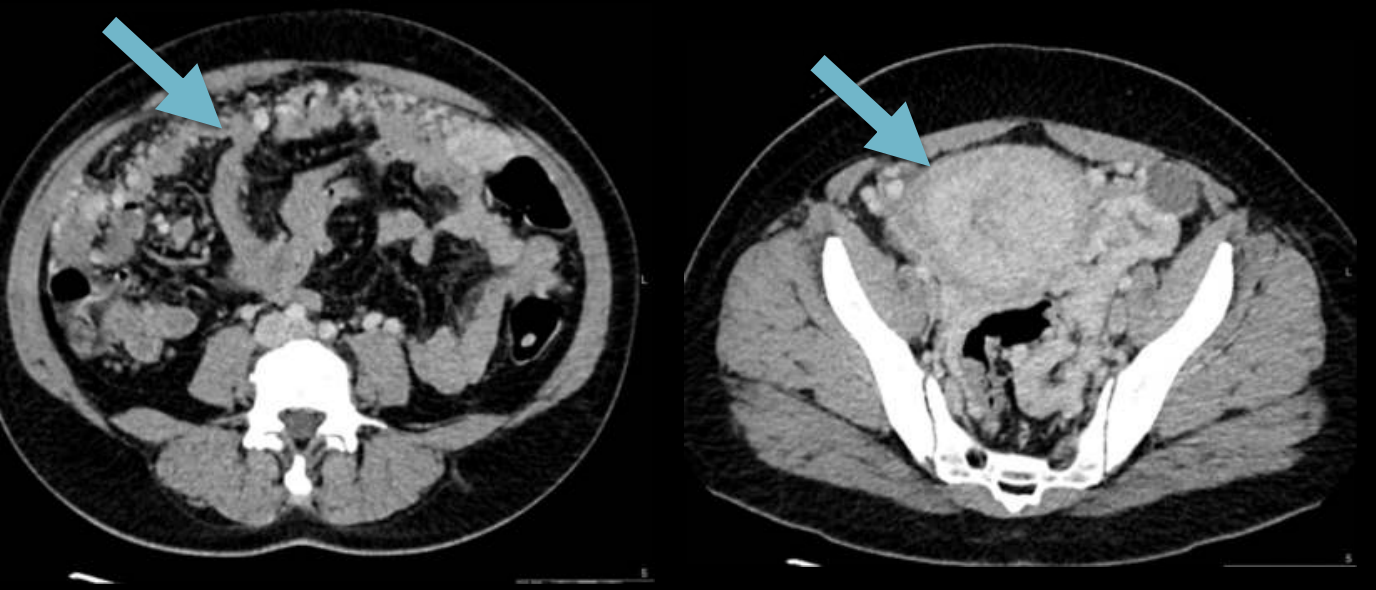

Axial CT scans from a 46 years old female patient with known diffuse peritoneal

leiomyomatosis. Note some nodule implants on mesentery and marked enlarged uterus

\section{Tuberculous Peritonitis}

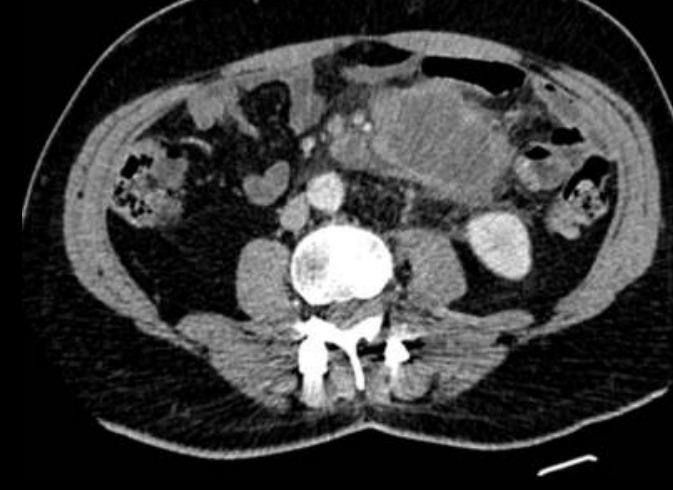

Patient with a kno adhering to the mesentery. Biopsy confirmed tuberculous peritonitis ("fixed fibrotic type").

\section{Conclusions} Invasive peritoneal disease includes more than just peritoneal carcinomatosis, and the radiologist should be aware of the main differential diagnosis to ensure a proper patient management. 